\title{
Serum lipids in young patients with ischaemic stroke: a case-control study
}

\author{
J F Albucher, J Ferrieres, J B Ruidavets, B Guiraud-Chaumeil, B P Perret, F Chollet
}

\begin{abstract}
Objectives-The relation betweem serum lipids and ischaemic stroke remains controversial. Studies of lipid related risk factors in cerebrovascular disease have varied greatly in their findings and also in their definition of the cerebrovascular end points. Serum lipids are thought to interact with the pathogenesis of stroke through an atherosclerosis mechanism. Stroke in young patients have been shown to be related to non-atherosclerotic causes most of the time. The aim was to determine the serum lipid profile and the vascular risk factors for ischaemic stroke in a series of patients under 45 with an ischaemic stroke and to compare them with a series of controls of the same age. Methods-Ninety four consecutive patients with ischaemic stroke were compared with 111 controls of the same age recruited from a regional electoral list. Vascular risk factors were recorded and serum lipid profile was determined in all of them.
\end{abstract}

Results-Multivariate analyses showed that low HDL cholesterol, male sex, smoking, hypertension, and oral contraceptives were risk factors for intracerebral arterial occlusion.

Conclusion-Low HDL cholesterol was the only serum lipid index to be associated to an increased risk of stroke in this population. Low HDL cholesterol must be considered in the care management of young patients regardless of the detectable presence of atherosclerosis.

(F Neurol Neurosurg Psychiatry 2000;69:29-33)

Keywords: stroke; young people; lipoproteins; HDL cholesterol; vascular risk factors

The relation between serum lipids and lipoproteins in ischaemic cerebrovascular disease is not clear as it is in coronary heart disease. ${ }^{1}$ The role of high concentrations of serum cholesterol as a risk factor for stroke is still undecided at the present time. ${ }^{2}$ Studies of lipid related risk factors in cerebrovascular disease have varied greatly in their findings and also in their definition of the cerebrovascular end points..$^{3-11}$

Several clinical trials showed an association between high concentrations of serum cholesterol and ischaemic stroke. ${ }^{12-15}$ On the other hand, case-control studies of stroke which examined cholesterol as a risk factor have generally produced negative findings ${ }^{16}{ }^{17}$ and prospective studies have generally failed to show a direct and strong association. ${ }^{18}$ Prospective
Studies Collaborators concluded from a review of 13000 strokes and 45 prospective cohorts that after standardisation for age, there was no association between blood cholesterol and stroke. ${ }^{19}$ However, others have shown the effects of cholesterol lowering treatment on the prevention of stroke. ${ }^{20-22}$ Recently, pravastatin was shown to reduce the risk of stroke in a population of patients with coronary disease. ${ }^{23}$

Several biases can have modified the results of studies and their interpretation: multiple aetiologies and stroke subtypes, ethnical influence, diagnosis criteria, and influence of the stroke on serum lipid concentrations. ${ }^{24} 25$ In most of the studies, high serum concentration of cholesterol was suspected to increase the risk of stroke through the genesis of atherosclerotic lesions. It seemed to us interesting to investigate serum lipids in a population of patients with arterial occlusion but with little atherosclerosis.

Ischaemic stroke in young patients has been extensively studied in the past few years. ${ }^{11}{ }^{26-31}$ It was shown in most of these series that atherosclerosis has little role in the mechanism of stroke.

The aim of our study was to select prospectively a series of consecutive patients under 45 who presented with an ischaemic stroke, to perform extensive aetiological investigations to evaluate the cause of the stroke as precisely as possible, and to compare their serum lipid and lipoprotein profiles with a control population of the same age.

\section{Patients and methods}

PATIENTS

From 1993 to 1997,94 consecutive patients aged 15 to 45 years ( 55 males, 39 females aged 35.8 (SD 8.2) years) admitted to the Department of Neurology of the Purpan Hospital in Toulouse (France) for acute ischaemic stroke were included in this study.

All of them presented a focal neurological deficit of sudden onset. Transient ischaemic attacks (complete regression of all neurological deficits in less than 24 hours) and strokes were both included.

Clinical examination by a neurologist and cerebral CT without contrast injection were performed in all of them after admission.

All the patients were asked for personal and familial medical history. Previous stroke, migraine, deep venous thrombosis, coronary heart disease, and foetal loss were recorded.

Extensive aetiological investigations were performed as follows:

In all patients ECG, chest radiography, blood chemistry, red and white blood cell counts, and erythrocyte sedimentation rate 
were evaluated. Immunological tests included immunoelectrophoresis, antinuclear antibodies, and antiphospholipid antibodies. Serological tests for syphilis and HIV were performed with the patients' consent.

Haemostatic laboratory investigations (including platelet count, activated partial thromboplastin time (aPTT), prothrombin time, plasma concentrations of fibrinogen, antithrombin III, C and S protein, lupus anticoagulant) were systematically performed before anticoagulant treatment.

Four vessel cerebral angiography was performed as soon as possible after admission (85 out of 94 patients). In comatose patients angiography was not performed. During the past year cerebral magnetic resonance angiography (MRA) was done in seven patients.

On the eighth day after admission, inpatients underwent cerebral CT with contrast injection or cerebral MRI was performed to assess brain infarct localisation.

Systematic cardiac evaluation included transthoracic (TTE) and transoesophageal (TEE) echocardiography. When patients were unable to swallow, TEE was not done. TEE was performed with a $5 \mathrm{MHz}$ multiplan probe. An intravenous saline contrast injection was given to all the subjects to search for any interatrial septum defect. Atrial septal aneurysm (ASA) was defined as a mobile zone of the fossa ovalis with an excursion of at least 15 $\mathrm{mm}$ into one of the atria during the cardiac cycle. Patent foramen ovale (PFO) was defined as an interatrial septum defect with appearance of bubbles in the left atrium within three cardiac cycles after brachial saline contrast injection.

Significant aortic atheroma was defined as a protrusion $>5 \mathrm{~mm}$ into the aortic lumen.

\section{Aetiology of strokes}

The aetiology of ischaemic stroke was attributed using TOAST classification criteria (table 1). ${ }^{32}$ Twenty three patients were found with cervical large vessel lesions, mainly arterial dissections. Stroke was considered of atheromatous origin when significant $(>70 \%)$ carotid stenosis was established on cerebral arteriography. Three patients only had a significant cerebral vessel atheroma. Lacunar infarction was defined as a typical neurological syndrome associated with a small subcortical infarct on CT or cerebral MRI. Cardioembolic strokes were classified into high risk and medium risk

Table 1 Aetiologies of ischaemic strokes (TOAST classification)

\begin{tabular}{ll}
\hline Large artery disease: $20(21.3 \%)$ & $\begin{array}{l}\text { Arterial dissection } \mathrm{n}=14 \\
\text { Moya-Moya disease } \mathrm{n}=3\end{array}$ \\
& $\begin{array}{l}\text { Significant carotid stenosis } \mathrm{n}=1 \\
\text { Intracranial atheromatosis } \mathrm{n}=1\end{array}$ \\
& $\begin{array}{l}\text { Significant aortic atherosclerosis } \mathrm{n}=1 \\
\text { High risk of embolism }: 15\end{array}$ \\
& $\begin{array}{l}\text { Medium risk of embolism }: 20 \\
(19 / 20 \text { with PFO and ASIA) }\end{array}$ \\
Cardiac embolism: $35(37.2 \%)$ & $\begin{array}{l}\text { Haemostatic disturbances }: 2 \\
\text { Antiphospholipid antibodies syndrome : } 1 \\
\text { Rendu-Osler disease }: 1\end{array}$ \\
Other cause of ischaemic stroke: $9(9.6 \%)$ & $\begin{array}{l}\text { Behçet's disease }: 1 \\
\text { Cerebral arteritis }: 3 \\
\text { Eclampsia }: 1\end{array}$ \\
& \\
\hline
\end{tabular}

of embolism according to the TOAST classification. Nineteen of our 20 patients with medium risk cardiopathy were found with septal abnormalities (PFO and ASA). Stroke was considered of undetermined cause if no aetiology was found despite complete investigations or if more than two causes were found (table 1).

\section{CONTROLS}

One hundred and eleven volunteers of the same age (36.8 (SD 0.98)) years, range 35-45) were randomly recruited during the same period from a survey of a random sample of those registered on the electoral rolls of the Toulouse region.

\section{VASCULAR RISK FACTORS}

Vascular risk factors such as cigarette smoking, arterial hypertension (systolic blood pressure $>160 \mathrm{~mm} \mathrm{Hg}$, and diastolic blood pressure $>90 \mathrm{~mm} \mathrm{Hg}$ ), hyperlipoproteinaemia (patients with total cholesterol $>5.7 \mathrm{mmol} / 1$ or LDL cholesterol $>3.5 \mathrm{mmol} / 1$ or with lowering cholesterol treatment), diabetes mellitus, and oral contraceptives were also reported for patients and controls.

\section{LIPOPROTEIN ANALYSIS}

Blood samples were obtained in the morning after an overnight fast for patients and controls. For patients, blood samples were taken in the morning after their admission. Some of the patients were already on heparin while blood samples were taken but in no cases did the duration of ongoing heparin treatment exceed 24 hours.

Venous blood samples were collected into EDTA tubes and were centrifuged at $4^{\circ} \mathrm{C}$ and $2500 \mathrm{~g}$ for 15 minutes. Serum total cholesterol was measured by the cholesterolesterase/ cholesterol oxidase technique using commercial kits (Boehringer, Mannheim, Germany). ${ }^{33}$ Likewise, triacylglycerols were measured enzymatically (Boehringer, Mannheim, Germany). ${ }^{34}$ HDL cholesterol was determined after precipitation of lower density lipoproteins by a phosphotungstic reagent (Boehringer, Mannheim, Germany). Interassay coefficients of variation were $2 \%$ for cholesterol and triacylglycerols and $8 \%$ for HDL cholesterol. Apolipoproteins AI and B were measured by first order immunoprecipitation (Cobas MIRA, Roche, Basel, Switzerland) with interassay coefficients of variation less than $5 \%$.

Lipoprotein particles Lp AI and Lp(a) were determined by rocket immunoelectrodiffusion using commercial plates (Sebia, Issy-lesMoulineaux, France). Coefficients of variation were $5 \%$ for $\mathrm{LpAI}$ and $10 \%$ for $\mathrm{Lp}(\mathrm{a}) .^{35}$

\section{STATISTICAL ANALYSIS}

Comparison between the two groups was made using Student's $t$ test for independent samples.

The relation between demographic, clinical, and biological indices and the presence or absence of cardiovascular disease was examined by multiple logistic regression. Likelihood ratio statistics were used to compare models with different combinations of predictors. Both 
Table 2 Lipoprotein concentrations in patients and controls

\begin{tabular}{llll}
\hline & $\begin{array}{l}\text { Control subjects } \\
(n=111)\end{array}$ & Patients $(n=94)$ & $p$ Value \\
\hline Cholesterol (mmol/l) & $5.53(1.03)$ & $5.7(1.2)$ & NS \\
HDL cholesterol (mmol/l) & $1.77(0.5)$ & $1.36(0.4)$ & 0.0001 \\
LDL cholesterol (mmol/l) & $3.26(1)$ & $3.66(1.1)$ & 0.0108 \\
VLDL cholesterol (mmol/l) & $0.48(0.24)$ & $0.63(0.34)$ & 0.0004 \\
Triglycerides (mmol/l) & $1.1(0.66)$ & $1.43(0.8)$ & 0.0014 \\
ApoA1 (g/l) & $1.61(0.23)$ & $1.49(0.28)$ & 0.0006 \\
ApoB $(\mathrm{g} / \mathrm{l})$ & $1.11(0.26)$ & $1.22(0.34)$ & $<0.05$ \\
Lp (a) $(\mathrm{g} / \mathrm{l})$ & $0.23(0.36)$ & $0.24(0.35)$ & $\mathrm{NS}$ \\
LPA1 $(\mathrm{g} / \mathrm{l})$ & $0.48(0.18)$ & $0.51(0.23)$ & $\mathrm{NS}$ \\
\hline
\end{tabular}

Values are mean (SD).

Table 3 Multivariate analysis

\begin{tabular}{lc}
\hline & $\begin{array}{l}\text { Odds ratio and 95\% } \\
\text { confidence intervals }\end{array}$ \\
\hline Age (per y) & $0.976(0.910-1.047)$ \\
Sex (male/female) & $3.15(1.22-8.13)$ \\
Smoking (current smokers/non-smokers) & $6.8(2.67-17.28)$ \\
Diabetes mellitus (diabetes/no diabetes) & $1.087(0.058-20.38)$ \\
Hypertension (hypertension/no hypertension) & $18.67(2.04-170.76)$ \\
Hyperliproteinemia (hyperliporoteinaemia/no hyperlipoproteinaemia) & $0.73(0.077-6.88)$ \\
Oral contraceptives (use of oral contraceptives/no oral contaraceptives) & $7.33(1.79-29.9)$ \\
Fibrinogen (per g/l) & $1.73(0.95-3.16)$ \\
HDL cholesterol (per mmol/l) & $0.174(0.067-0.45)$ \\
\hline
\end{tabular}

Degrees of freedom $=9$ and $-2 \log$-likelihood $=170.62$.

forward stepwise and backward stepwise trait selection procedures were used to identify a parsimonious set of predictors. Forward stepwise trait selection was also carried out to identify pairwise interactions. The predictors in the simplest adequate model, identified by the stepwise procedure, were then evaluated by the likehood ratio statistical test with a significance level of 0.05 .

\section{Results}

SINGLE VARIABLE ANALYSIS

Serum lipid and apolipoprotein concentrations

Total serum cholesterol concentrations, LpA1, and $\mathrm{Lp}$ (a) did not differ between patients and controls whereas LDL cholesterol, VLDL cholesterol, ApoB, and triglycerides were significantly higher in patients than in controls. HDL cholesterol and ApoA1 were significantly lower in patients (table 2). Six of our 94 patients presented a previously known hyperlipoproteinaemia whereas only two the 111 controls did.

Other vascular risk factors and ischaemic stroke Hypertension - Twelve of our 94 patients showed pre-existing hypertension (12.7\%) compared with only two of the 111 controls $(1.8 \%)$.

Smoking-At the moment of the stroke 57 of our 94 patients $(60.6 \%)$ were smokers whereas only 31 of 111 controls $(27.9 \%)$ were recorded as smokers $(\mathrm{p}<0.001)$.

Diabetes mellitus - A very low incidence of diabetes was found in our population of patients $(3 / 94 ; 3.2 \%)$ that was not different from controls.

Oral contraceptives-Eighteen of the 39 women from our series of patients with stroke were taking oral contraceptives $(46.1 \%)$ but only six from 49 in the control group $(12.2 \%)$ $(\mathrm{p}<0.01)$.

Fibrinogen-Serum fibrinogen concentration was significantly higher in patients (3.13 (SD 1) $\mathrm{g} / \mathrm{l})$ than in controls (2.67 (SD 0.52) g/l) $(\mathrm{p}<0.001)$.
Multivariable analysis

Multivariable analysis showed that among all lipid indices HDL cholesterol was the only one to be highly associated with stroke as an independent variable. Sex (risk higher in men) smoking habit, hypertension, and oral contraceptives in women can also be recognised as independent variables positively associated with stroke in our series (table 3 ).

\section{Discussion}

We chose to study serum lipids and lipoproteins in a highly selected population of young patients (under 45) with stroke. Stroke in young patients is a relatively rare event but it has been extensively studied in the past few years. ${ }^{11}{ }^{26-31}$ The causes of stroke of the young are multiple (table 1) but a low rate of atherosclerosis is recognised in the genesis of stroke. The incidence of demonstrated atherosclerosis varies from $5 \%{ }^{26}$ to $17 \%{ }^{27}$ of the patients. In our series atherosclerosis was detected in three of our 94 patients only (3.2\%). This aspect represents a major difference with the global population of patients with stroke and makes stroke in young people a remarkable "model" of arterial occlusion with very few clinically significant atherosclerosis components.

In our study, the blood samples were taken from the patients at the very beginning of the hospital stay (within the first 24 hours) most of the time before heparin administration, after 12 hours fasting to avoid any effects of the hospital diet. Butterworth et al showed that changes in serum lipids were found after a stroke. Total cholesterol, LDL, and HDL cholesterol significantly fell during the first week after stroke although the concentrations normalised after 3 months. ${ }^{36}$ We think that in our study, blood samples were taken early enough to prevent any false negative interpretation of our data.

Conflicting results exist in the literature about the correlation between the total plasma cholesterol of patients and the risk of stroke. ${ }^{2}$ Case-control studies examining total cholesterol as a risk factor have generally produced negative findings. ${ }^{16}$ However, some clinical observational studies found positive correlation. $^{12-15}$ In an overview of 10 prospective studies examining the relation between total cholesterol and subsequent stroke, Qizilbash et al concluded that there was a significant association. ${ }^{24}$ Other studies were less conclusive. ${ }^{19}$ However, when patients under 45 were screened total cholesterol was associated with an increased risk of stroke. The Copenhagen City Heart Study ${ }^{18}$ showed a non-log linear pattern between plasma cholesterol and the risk of ischaemic cerebrovascular disease. The risk was confined to the upper $5 \%$ of the cholesterol distribution.

On the other hand, there are several arguments in the literature for the effects of cholesterol lowering treatment on the prevention of stroke. The Scandinavian Simvastatin Survival Study ${ }^{20}$ provides evidence for the beneficial effect of cholesterol lowering on fatal and non-fatal cerebrovascular events. Even if the results of cholesterol-lowering trials on fatal 
and non-fatal stroke which have been investigated in two reviews ${ }^{21} 22$ reached different conclusions, it must be mentioned that more recent meta-analysis including trials with inhibitors of HMGCoA reductase suggested an actual reduction of stroke risk in patients with coronary heart disease of about $25 \% .{ }^{37}{ }^{38}$ More recently, the CARE trial showed a strong reduction in the occurrence of stroke in patients with coronary heart disease with a daily dose of $40 \mathrm{mg}$ pravastatin. ${ }^{23}$

Many reasons have been proposed to account for such discrepancies: errors in the clinical diagnosis of stroke (absence of CT), ethnic influence, multiple aetiologies, ${ }^{25} 39$ and subtypes of stroke. ${ }^{40}$ In our study, total plasma cholesterol was similar in the group of patients and the controls. This confirms that total plasma cholesterol when normal does not guarantee the absence of lipid risk factor for a stroke in young people. Our patients had lower HDL cholesterol and ApoA1 than controls. We found that HDL cholesterol was the only independent serum lipid factor for stroke in young people in this study. Low HDL cholesterol concentration in patients with cerebrovascular disease has been described in prospective studies ${ }^{718}$ and in case-control studies. ${ }^{94142} \mathrm{Few}$ found no association. ${ }^{43}$ Moreover, some arguments now appear in the literature showing that drug induced increases of HDL cholestrol led to a reduced rate of progression of coronary atherosclerosis with no change in the plasma LDL cholesterol concentration ${ }^{44}$ These findings were correlated with current conceptions of atherosclerosis ${ }^{45}$ that HDL cholesterol is responsible for the reverse transport of cholesterol from tissues to the liver for bile formation and prevents atherogenesis. Atherosclerosis is considered to be the main pathology underlying ischaemic stroke as well as myocardial infarction. The generally accepted mechanism of action of lipids in causing ischaemic stroke relies on immediate precursors of arterial occlusion: arterial stenosis, arterial embolus, small vessel disease, etc. In our case, advanced and detectable atherosclerosis cannot be significantly implicated in stroke genesis in most of the subjects. So, we suggest that low HDL cholesterol is a risk factor for stroke in young people through other mechanisms. Several arguments can be found in the literature to show the role of HDL in arterial wall metabolism. Interactions between LDL particles, vascular smooth muscle cells, endothelial cells, and HDL particles have been described. LDL induced cytotoxicity against human vascular smooth muscle cells and endothelial cells was inhibited by the presence of HDL. ${ }^{46}$ So, it can be postulated that low HDL concentration gives reduced protection against LDL toxicity. Moreover, more recently, an HDL associated paraoxonase was proved to give protection against LDL oxidative modifications. ${ }^{47} 48$ These complex interactions might participate in very early phases of atherosclerosis development and it might be that in our patients, the low HDL concentration participates in early preclinical developement of atherosclerosis.
Controversies also exist about the association between concentrations of serum triglycerides and the risk of stroke. Some studies led to negative results ${ }^{6} 1249$ whereas others showed a positive association ${ }^{95}$ between the risk of stroke and high serum triglyceride concentrations. In our study no correlation was found and it seems that high plasma triglyceride concentration cannot be recognised as a risk factor of stroke in young people. More surprising, in our study, was the absence of association between $\mathrm{Lp}(\mathrm{a})$ concentration and stroke. High concentrations of $\operatorname{Lp}(a)$ have been found to be associated with ischaemic stroke in many studies. ${ }^{51-55}$ Several potential reasons can be proposed to explain the difference between our negative data and some of the series in the literature. Firstly, the aetiologies of stroke were perhaps different and related to a variety of mechanisms. Secondly, there was, in all the studies, a very large distribution of liporotein (a) values: SDs were high in our series and in others. This suggests a two peak distribution of $\mathrm{Lp}$ (a) values. We could not confirm this hypothesis. In our study a very low rate of atherosclerosis was detected (3.2\%). This is a major difference from other studies. From our study, we think that $\mathrm{Lp}$ (a) can be considered as a risk factor of ischaemic atherothrombotic stroke but not as a risk factor for strokes of other origins.

Sex, smoking, and hypertension were found to be independent risk factors for stroke in our series. This is in agreement with published data. Oral contraceptives were also found to be an independent risk factor for stroke. Differences can be found between American and European studies about the risk of myocardial infarction and stroke in women taking oral contraceptives. European studies showed oral contraceptive use in general to be associated with an increased risk of ischaemic stroke and myocardial infarction whereas the United States database does not confirm a direct relation between contraceptive use and arterial risk. ${ }^{56}$ It is accepted that recent low dose oral contraceptives only have a very moderate effect on arterial risk. ${ }^{58}$ We have no information about the actual dose of ethinyl estradiol in our patients so it is impossible to relate the increased risk to a second or third generation category of oral contraceptive. However, from our data, oral contraceptives must still be considered as a risk factor for stroke.

We clearly showed risk factors for stroke in young people in this study. HDL cholesterol concentration seems the only serum lipid index which can be independently associated with an increased risk of stroke. Total cholesterol seems to be poorly associated. The association between low HDL cholesterol and risk of stroke was very strong and HDL cholesterol remains the strongest risk factor. It is also important to notice that the evidenced risk factors for stroke in our study probably interacted with the arterial occlusion through nonatherosclerosis mechanisms as atherosclerosis was rare in our population of patients. They can be considered more as risk factors for intracerebral arterial occlusion than as risk fac- 
tors for atherothrombotic events. We think that HDL cholesterol should be systematically considered in our patients' assessement regardless of the detectable presence of arterial atherosclerosis lesions.

1 Tell GS, Crouse JR, Furberg CD. Relation between blood lipids, lipoproteins, and cerebrovascular atherosclerosis. A review. Stroke 1988;19:423-30.

2 Postiglione A, Napoli C. Hyperlipidaemia and atherosclerotic cerebrovascular disease. Curr Opin Lipidol 1995;6: 236-42.

3 Heyman A, Nefzger MD, Estes EH. Serum cholesterol level in cerebral infarction. Arch Neurol 1961;5:264-8.

4 Feldman RG Albrink. MJ. Serum lipids and cerebrovascular disease. Arch Neurol 1964;10:91-100.

5 Kannel WB, Gordon T, Dawber TR. Role of lipids in the development of brain infarction: the Framingham study Stroke 1974;5:679-85.

6 Rossner S, Kjellin K, Mettinger KL, et al. Normal serum cholesterol but low HDL-cholesterol concentrations in young patients with ischemic cerebrovascular disease. Lancet 1978;i:577-9.

7 Sirtori CR, Gianfranceschi G, Gritti I, et al. Deceased high density lipoprotein cholesterol in male patients with transient ischaemic attack. Atherosclerosis 1979;32:205-11.

8 Taggart H, Stout RW. Reduced high density lipoprotein in stroke: relationship with elevated triglyceride and hyperstroke: relationship with elevated triglyceri
tension. Eur 7 Clin Invest 1979;9:219-21.

9 Nubiola AR, Massana I, Masdeu S, et al. High density lioprotein cholesterol in cerebrovascular disease. Arch Neurol 1981;38:468.

10 Murai A, TanakaT, Miyahara T, et al. Lipoprotein abnormalities in the pathogenesis of cerebral infarction and abnormalities in the pathogenesi

11 Adams HP, Kappelle LJ, Biller J, et al. Ischemic stroke in young adults. Experience in 329 patients enrolled in the Iowa registry of stroke in young adults. Arch Neurol 1995;52:491-5.

12 Wolf PA, Kannel WB, Dawber TR. Prospective investigation: the Framingham study and the epidemiology of stroke. Adv Neurol 1978;19:107-20.

13 Frank JW, Reed DM, Grove JS, et al. will lowering population level of serum cholesterol affect total mortality. 7 Clin Epidemiol 1992;45:333-46.

14 Iso $\mathrm{H}$, Jacobs D, Wentworth D, et al. Serum cholesterol levels and 6 year mortality from stroke in 350977 men screened from the multiple risk factor intervention trial. $N$ Engl f Med 1989;320:904-10.

15 Westlund K, Nicolaysen R. Ten year mortality and morbidity related to serum cholesterol. If Clin Lab Invest ity related to serum

16 Noma A, MatsushitaS, Komori T. High and low density lipoprotein cholesterol in myocardial infarction and cerebral infarction. Atherosclerosis 1979;32:327-31.

17 Tilvis RS, Rekinjuntti T, Sulkava R, et al. Serum lipids and fatty acids in ischaemic strokes. Am Heart f 1987;113:61519.

18 Lindenstrom E, Boysen G, Nyboe J. Influence of total cholesterol, high density lipoprotein cholesterol, and triglyceride on risk of cerebrovascular disease. BMF 1994;309:1115.

19 Prospective Studies Collaboration. Cholesterol, diastolic blood pressure, and stroke: 13000 strokes in 450000 people in 45 prospective cohorts. Lancet 1995;346:1647-53.

20 Pedersen TR, Kjekshus J, Berg K, et al. randomized trial of cholesterol lowering in 4444 patients with coronary heart disease: the Scandinavian simvastatin stroke study. Lancet 1994;344:1383-9.

21 Atkins D, Bruce M, Psaty BM, et al. Cholesterol reduction and the risk for stroke in man. Ann Intern Med 1993;119:136-45.

22 Hebert PR, Gaziano M, Hennekens CH. An overview of trials of cholesterol lowering and risk for stroke. Arch Intern Med 1995;155:50-5.

23 Plehn JF, Davis BR, Sacks FM, et al. Reduction of stroke incidence after myocardial infarction, with pravastatin: the cholesterol and recurrent events study (CARE). Circulation 1999;99:216-23.

24 Qizilbash N, Duffy SW, Warlow C, et al. Lipids are risk factors for ischaemic stroke: overview and review. Cerebrovasc Dis $1992 ; 2: 127-36$

25 Qizilbash N. Are risks factors for stroke and coronary disease the same? Curr Opin Lipidol 1998;9:325-8.

26 Bogousslavsky J, Pierre P. Ischemic stroke in patients under age 45. Neurol Clin 1992;10:113-24.

27 Lisovoski F, Rousseaux P. Cerebral infarction in young people. A study of 148 patients with early cerebral angiople. A study of 148 patients with early cerebral an
graphy. $\mathcal{F}$ Neurol Neurosurg Psychiatry 1991;54:576-9.

28 Gautier JC, Pradat-Diehl P, Loron P, et al. Accidents vasculaires cérébraux des sujets jeunes. Une étude de 133 patients agés de 9 à 45 ans. Rev Neurol (Paris) 1989;145:437-42.

29 Rouhart F, Zagnoli F, Goas JY, et al. Accidents artériels ischémiques cérébraux de l'adulte jeune. 40 cas. Rev Neu rol (Paris) 1993;149:547-53.
30 Van den Berg JSP, Limburg M. Ischemic stroke in the young: influence of diagnostic criteria. Cerebrovasc Dis 1993;3:227-30

31 Woimant F, Rougemont J, Gardettte J, et al. Facteurs de risque vasculaire des accidents ischémiques cérébraux des sujets agés de 45 ans ou moins. Etude prospective de 200 patients. In: Bès A, Bounhoure JP, Geraud G, eds. Coeur et Cerveau. Paris: Masson, 1994:112-16.

32 Adams HP, Bendixen BH, Kappelle LJ, et al. Classification of subtype of acute ischemic stoke. Definitions for use in a multicenter clinical trial. Stroke 1993;24:35-41.

33 Roschlau P, Bernet, E, Gruber N. Enzymatische bestimmung des gesamchlesterins im serum. Z Klin Chem Klin Biochem 1974;12:403-7.

34 Wahefeld A. Triglyceride determination after enzymatic hydrolysis. Method of enzymatic analysis. 2nd ed. Weinheim: Verlag Chimie, 1974:1131-5.

35 Parra HJ, Arveiller D, Evans A. A case-control study of lipoprotein particles in two population at contrasting risk for coronary heart disease. Arteriosclerosis and Thrombosis 1992;12:701-7.

36 Butterworth RJ, Marshall WJ, Bath PMW. Changes in serum lipid measurements following acute stroke. Cerebrovac Dis 1997;7:10-13.

37 Hebert P, Gaziano, JM, Hennekens CH. Cholesterol lowering with statin drugs, risk of stroke and total mortality. FAMA 1997;278:313-21.

38 Bucher H, Griffith LE, Guyatt GH. Effect of HMGcoa reductase inhibitors on stroke. A meta-analysis of randomised controlled trials. Ann Intern Med 1998;128:89-95.

39 Hachinski V, Graffagnino C, Beaudry M, et al. Lipids and stroke: a paradox resolved. Arch Neurol 1996;53:303-8.

40 Lindgren A, Nilson-Ehle P, Norrving B, et al. Plasma lipids abd lipoproteins in subtypes of stroke. Acta Neurol Scand 1992;86:572-8.

41 Qizilbash N, Jones L, Warlow C, et al. Fibrinogen and lipid concentrations as risk factors for transient ischaemic attacks and minor ischaemic strokes. BMF 1991;303:605-

42 Sidharam R. Risk factors for ischaemic stroke: a case control analysis. Neuroepidemiology 1992;11:24-30.

43 Aronow WS, Gutstein H, Lee NH, et al. Three-year follow up of risk factors correlated with new atherothrombotic brain infarction in 708 elderly patients. Angiology 1988;39: $563-6$.

44 Ericsson C. Results of the bezafibrate coronary atherosclerosis intervention trial (BECAIT) and an update of trials now in progress. Eur Heart $\mathcal{F}$ 1998;19:H37-41.

45 Yatsu FM, Fischer M. Atherosclerosis: current concepts on pathogenesis and interventional therapies. Ann Neurol 1989;26:3-12.

46 Hessler J, Lazzarini-Robertson A, Chisolm GM. LDLinduced cytotoxicity and its inhibition by HDL in human vascular smooth muscle and endothelial cells in culture. Atherosclerosis 1979;32:213-29.

47 Mackness M, Arrol S, Abbott C, et al. Protection of LDL against oxydative modification by HDL associated paraoxonase. Atherosclerosis 1993;104:129-35.

48 Kelso G, Stuart WD, Richter RJ, et al. Apolipoprotein J is associated with paraoxonase in human plasma. Biochemistry 1994;33:832-9.

49 Smith GDSM, Marmot MG, Rose G. Plasma cholesterol concentrations and mortality. $\mathscr{7} A M A$ 1992;267:70-6.

50 Ronbinson RW, Higano N, Cohen WD. Comparison of serum lipid levels in patients with cerebral thrombosis and in normal subjects. Ann Intern Med 1963;59:180-5.

51 Zenker G, Költringer P, Boné G, et al. Lipoprotein(a) as a strong indicator for cerebrovascular disease. Stroke 1986; 17:942-6.

52 Murai A, Miyahara T, Fujimoto N, et al. Lp(a) Lipoprotein as a risk factor for coronary heart disease and cerebral infarction. Atherosclerosis 1986;59:199-204.

53 Jürgens G, Költringer P. Lipoprotein(a) in ischemic cerebrovascular disease: a new approach to the

54 Woo J LE, LamCWK, Kay R, et al. Hypertension, lipoprotein (a) and apolipoprotein A1 as risk factors for stroke in the Chinese. Stroke 1991;22:203-8.

55 Nagayama MSY, Nagayama T. Lipoprotein(a) and ischemic cerebrovascular disease in young adults. Stroke 1994;25: $74-8$.

56 Lewis M. Myocardial infarction and stroke in young women: what is the impact of oral contraceptive? $A m \mathcal{F}$ Obstet Gynecol 1998;179:S68-77.

57 Lidegaard $\mathrm{O}$. Thrombotic diseases in young women and the influence of oral contraceptives. Am f Obstet Gynecol 1998; 179:S62-7.

58 Mant J, Painter R, Vessey M. Risk of myocardial infarction, angina and stroke in users of oral contraceptives: an updated analysis of a cohort study. Br $\mathcal{F}$ Obstet Gynaecol 1998; 105:890-6.

59 Van Lunsen H. Recent oral contraceptive use patterns in four European countries: evidence for selective prescribing of oral contraceptives containing third-generation progestogens. European fournal of Contraception and Reproductive Health Care 1996;1:39-45. 